\title{
Design and locomotion analysis of two kinds of rolling expandable mobile linkages with a single degree of freedom
}

(C) The Author(s) 2020. This article is published with open access at link.springer.com and journal.hep.com.cn

\begin{abstract}
This study presents two kinds of rolling robots that are able to roll by deforming their outer shapes with a single degree of freedom. Each robot is an essential multiloop planar expandable linkage constructed by a concave outer loop and several inner parallelogram loops. In this study, the mechanical design of the robots is introduced. Dynamic rolling process is further analyzed on the basis of zero moment point method, and a morphing strategy is proposed to guarantee a stable dynamic rolling process. A novel passive rolling locomotion is also developed, which enables the robots to roll and stand on a slope. To verify the design, two prototypes are manufactured, wherein the dynamic and passive rolling locomotion are carried out.
\end{abstract}

Keywords rolling locomotion, expandable mechanism, mechanism design, zero moment point (ZMP) analysis

\section{Introduction}

Rolling is one of the fundamental locomotion modes in nature, which demonstrates passive and active rolling modes [1]. Some organisms can perform rolling locomotion by deforming the shape of their bodies. For example, the Namib Golden Wheel Spider [2], and the Stomatopod shrimp [3] can deform their bodies into a cylindrical shape for ease of rolling. Inspired by these organisms, several

Received November 1, 2019; accepted January 5, 2020

Yanlin HAO, Yezhuo LI, Yan-An YAO

School of Mechanical, Electronic and Control Engineering, Beijing Jiaotong University, Beijing 100044, China

Yaobin TIAN $(\bowtie)$

School of Mechanical Engineering and Automation, Beihang University, Beijing 100191, China

E-mail: ybtian@buaa.edu.cn

Jianxu WU

Department of Mechanical Engineering, Tsinghua University, Beijing 100084, China mechanisms with looped configurations have been used for designing rolling robots. To generate stable and fast rolling locomotion, different morphing strategies have been developed, such as center of gravity (COG) offset [4], central pattern generators $[5,6]$, and zero moment point (ZMP) methods [7,8].

One of the typical rolling robots is constructed by a planar linkage with a single-loop configuration. For example, Liu et al. [8] used a four-bar linkage to design a rolling robot with a parallelogram shape. The ZMP method was used to control the robot to roll along a straight line. Yamawaki et al. [9] and Wang et al. [10] used a fivebar linkage to perform rolling locomotion with a pentagonal loop, and dynamic rolling process was implemented on the basis of COG offset method. By using several active revolute (R) joint modules, Sastra et al. [11] presented a modular method that can build a class of rolling robots with a polyhedral loop. A morphing strategy was proposed to change the shape of the loop. The major advantages of such single-loop rolling robot are as follows. First, the robot can perform a fast rolling process in a narrow space. Second, the mechanical structures are simple and easy to manufacture and assemble. Given that all motors, batteries, or controllers may be mounted along the loop, the fluctuation of motors' positions is large when the loop is rolling on the ground. The stiffness of such single-loop robot is weak. Some trade-offs between the deformation capability and the numbers of degree of freedom (DOF) are also observed.

To improve the rolling capability and stiffness of the robot, we develop a new kind of rolling robots with a single DOF on the basis of expandable linkages. These linkages contain a single outer loop with a concave polygonal shape, and several inner loops each of which retains a parallelogram shape. The outer loop enables the robot to roll on the ground. Motors, batteries, controllers, and other devices can be mounted at the center of the robot. Rolling locomotion is analyzed on the basis of ZMP method. Active and passive rolling modes are presented. A series of rolling experiments will be carried out on two 
prototypes to verify our design. The major advantages of our design are as follows: 1) Our design will protect the inner devices away from the ground; 2) the position of these devices will be changed smoothly when the robot rolls on the ground; and 3) owing to the multi-loop configuration, stiffness can also be enhanced.

The rest of this paper is organized as follows. Section 2 introduces the mechanical design of the rolling robot. Section 3 analyzes the dynamic rolling mode via ZMP method. Section 4 proposes the passive rolling mode on a slope. Section 5 presents the locomotion experiments on the basis of manufactured prototypes. Section 6 concludes the study.

\section{Mechanical design}

According to some planar expandable mechanism with a single DOF [12-14], the mechanisms of our robots are shown in Fig. 1. Each mechanism contains an outer loop and several inner parallelogram loops. Given that the shape of the outer loop can be determined by the number of the inner loops, for convenience, our robot is named as $n$-angular robot (wherein $n$ is the number of inner parallelogram loops). As shown in Fig. 1(a), the sixangular robot is composed of six equal $\mathrm{V}$-shaped links and two equal 3-branch links connected by $13 \mathrm{R}$ joint pairs. The 3D model of the mechanism is shown in Fig. 1(c), wherein the motor, battery, and controller are mounted in the middle of the robot. Similarly, as shown in Figs. 1(b) and 1(d), the second one is called an eight-angular robot, which contains eight V-shaped links, two 4-branch links, and $17 \mathrm{R}$ joints. The angles of $\mathrm{V}$-shape links of two robots are $120^{\circ}$ and $90^{\circ}$. The edge of each parallelogram is the same.

The mobility of our mechanism satisfies the GrübnerKutzbach mobility condition [15], and can be calculated as follows:

$$
M=3(n-1)-\sum_{i=1}^{g}\left(6-f_{i}\right),
$$

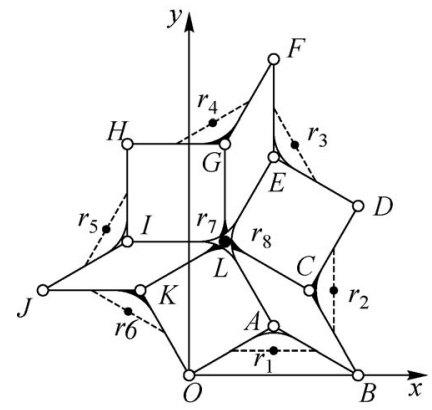

(a)

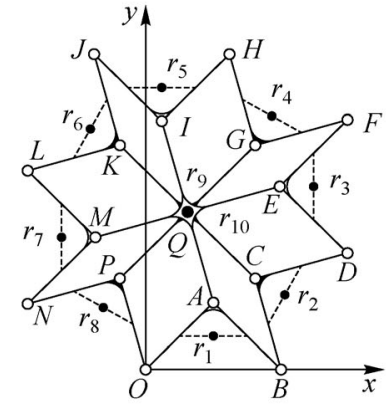

(b)

where $n$ is the number of links, $g$ is the number of joints, and $f_{i}$ is the DOF of the $i$ th joint.

Generally, the order of a planar mechanism is three. However, our mechanisms are composed of several parallelogram mechanisms. The order of our mechanisms is considered two. In this case, Eq. (1) can be further rewritten as

$$
M=2(n-1)-g .
$$

Substituting the numbers of the mechanism, the DOF of our robots (denoted by $M_{1}$ and $M_{2}$ ) can be determined as

$$
\left\{\begin{array}{l}
M_{1}=2 \times(8-1)-13=1, \\
M_{2}=2 \times(10-1)-17=1 .
\end{array}\right.
$$

Therefore, these mechanisms only have a single DOF. This result can also be obtained from the shape of the loops. Once the angle of a parallelogram is given, all shapes of the loop will be determined.

\section{Dynamic rolling locomotion analysis}

In our previous work [8], we used ZMP method to analyze the rolling locomotion of a parallelogram mechanism. The continually rolling process is realized by keeping the ZMP of the robot out of its supporting area. Referring to Fig. 2, let $\theta$ denote one angle in a parallelogram, whereas let $O, B$, $D, F, H$, and $J$ denote the six outer points, respectively. The basic rolling cycle of the six-angle robot can be described as follows.

(a) The initial state of the robot is a symmetric state with all parallelogram loops having the same shape, and $\theta=$ $60^{\circ}$.

(b) The critical tumbling position: By decreasing $\theta$, the ZMP of the robot will be moving out of its supporting area (line $O B$ ), and the robot will tumble about point $B$ to the right side.

(c) The tumbling motion: The robot will complete a tumbling motion about point $B$ that leads to a new link $(B D)$, thus coming into contact with the ground.

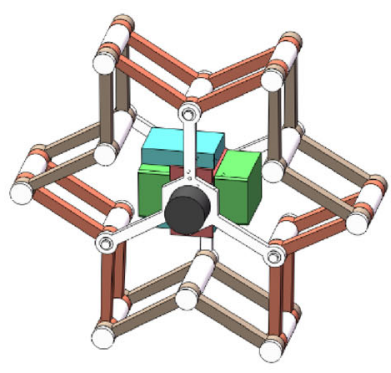

(c)

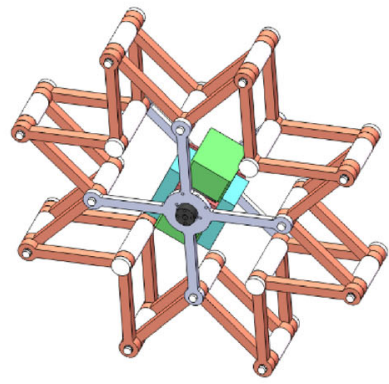

(d)

Fig. 1 Mechanism design of the proposed rolling robots: The sketches of (a) six- and (b) eight-angular robots; the corresponding CAD models of (c) six- and (d) eight-angular robots. 


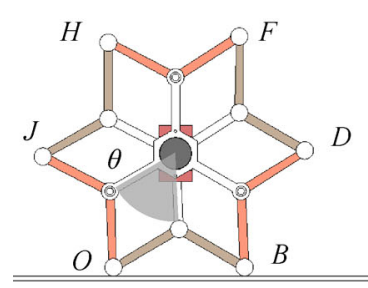

(a)

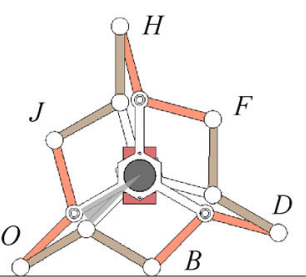

(b)

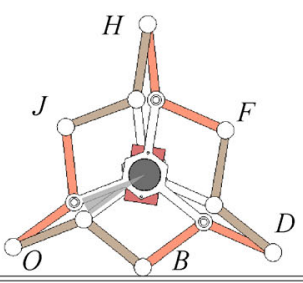

(c)

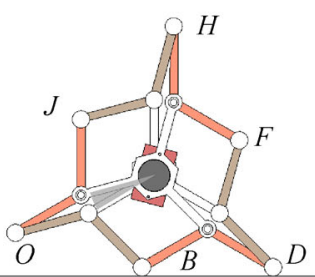

(d)

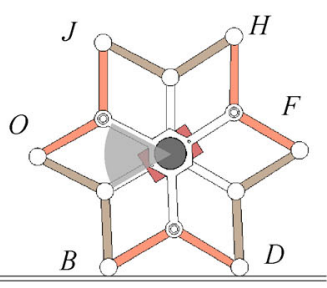

(e)

Fig. 2 Active rolling gait to the right side. (a) The initial state; (b) about to tumble; (c) the tumbling state; (d) after the tumbling motion; and (e) the recovery state.

(d) The recovering motion: After the tumbling motion, by increasing $\theta$, the robot begins to recover to the state that is similar to the initial state.

(e) The recovery state: At this moment, $\theta=60^{\circ}$, the robot has the same configuration with the initial state which is ready to perform the next rolling cycle.

The key process to generate the rolling locomotion is Step (b), given that the ZMP needs to be out of the supporting area. In the next section, the rolling condition and capability will be discussed by analyzing the ZMP at this state.

\subsection{Rolling conditions}

As shown in Fig. 3, two star-links are denoted by $L-A E I$ and $L-C G K$, and the six V-links are denoted by $O A B, B C D$, $D E F, F G H, H I J$, and $J K O$, respectively. The coordinate system is set at point $O$. Let $\boldsymbol{r}_{i}$ be the centroid of the $i$ th link, $m_{1}$ and $m_{2}$ are the mass of V-link and branch-link, respectively. Let $\theta$ be the input angle, i.e., the angle of $A L$ and $L K$. Let $\omega$ and $\alpha$ be the angular velocity and acceleration of $\theta$, respectively. Given that the mechanism is a planar mechanism, we only consider the component of ZMP in the $y$-axis (denoted by $y_{\mathrm{zmp}}$ ). According to the ZMP equations provided by Refs. [16,17], the $y_{\text {zmp }}$ can be expressed as follows:

$$
y_{\mathrm{zmp}}==\frac{\sum_{i=1}^{n}\left[m_{i} y_{i}\left(\ddot{z}_{i}+g_{z}\right)-m_{i} z_{i} \ddot{y}_{i}\right]}{\sum_{i=1}^{n} m_{i}\left(\ddot{z}_{i}+g_{z}\right)}
$$

where $m_{i}$ is the mass of link $i, \ddot{x}, \ddot{y}$, and $\ddot{z}$ are the accelerations along $x$-, $y$-, and $z$-axes respectively, $g_{z}$ is the acceleration of gravity, and $\boldsymbol{r}_{i}=\left[\begin{array}{ll}x_{i} & y_{i} z_{i}\end{array}\right]^{\mathrm{T}}$ is the center of mass of link $i$.

Then, the rolling condition of six-angular robot can be given as follows:

$$
\left\{\begin{array}{l}
y_{\mathrm{zmp}}<0 \text { (to the left side) } \\
y_{\mathrm{zmp}}>l \sqrt{3} \text { (to the right side). }
\end{array}\right.
$$

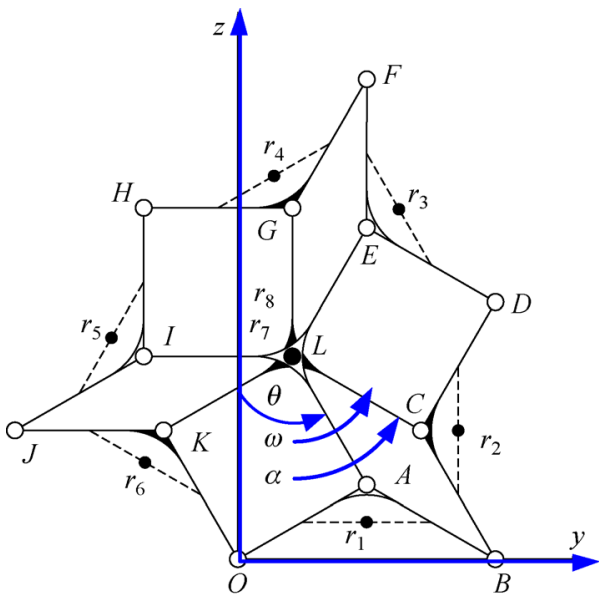

Fig. 3 ZMP analysis of six-angular robot.

\subsection{Rolling capability analysis}

According to the structure, the positions of points $O, A$, and $B$ can be expressed as follows:

$$
\begin{gathered}
\boldsymbol{r}_{O}=\left[\begin{array}{lll}
0 & 0 & 0
\end{array}\right]^{\mathrm{T}}, \boldsymbol{r}_{A}=\left[\begin{array}{lll}
0 & l \kappa & l \lambda
\end{array}\right]^{\mathrm{T}}, \\
\boldsymbol{r}_{B}=\left[\begin{array}{lll}
0 & 2 l \kappa & 0
\end{array}\right]^{\mathrm{T}},
\end{gathered}
$$

where $\kappa=\cos 30^{\circ}$ and $\lambda=\sin 30^{\circ}$.

For convenience, let $\beta_{1}=\theta+30^{\circ}$ and $\beta_{2}=\theta-30^{\circ}$. The positions of points $C, D, E, F, G, H, I, J, K$, and $L$ can be expressed in the terms of $\beta_{1}$ and $\beta_{2}$. Based on the positions of each points, the centroid of each link can be further obtained as follows:

$$
\boldsymbol{r}_{1}=\left[\begin{array}{lll}
0 & l \kappa & \frac{1}{2} l \lambda
\end{array}\right]^{\mathrm{T}}
$$

$\boldsymbol{r}_{2}=\left[\begin{array}{lll}0 & 2 l \kappa+\frac{l}{4}\left(3 \cos \beta_{1}+\cos \beta_{2}\right) & \frac{l}{4}\left(3 \sin \beta_{1}+\sin \beta_{2}\right)\end{array}\right]^{\mathrm{T}}$, 


$$
\begin{aligned}
& \boldsymbol{r}_{3}=\left[\begin{array}{ll}
0 & \frac{5}{4} l \kappa+l\left(\cos \beta_{1}+\cos \beta_{2}\right)
\end{array}\right. \\
& \left.\frac{3}{4} l \lambda+l\left(\sin \beta_{1}+\sin \beta_{2}+\frac{1}{2}\right)\right]^{\mathrm{T}},
\end{aligned}
$$

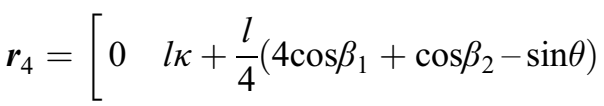

$$
\begin{aligned}
& \left.l \lambda+\frac{l}{4}\left(4 \sin \beta_{1}+\sin \beta_{2}-\cos \theta+3\right)\right]^{\mathrm{T}}, \\
& \boldsymbol{r}_{5}=\left[\begin{array}{ll}
0 & \frac{3}{4} l \kappa+l\left(\cos \beta_{1}-\sin \theta\right)
\end{array}\right. \\
& \left.\frac{3}{4} l \lambda+l\left(\sin \beta_{1}-\cos \theta+\frac{1}{4}\right)\right]^{\mathrm{T}}, \\
& \boldsymbol{r}_{6}=\left[\begin{array}{lll}
0 & \frac{l}{4}\left(3 \sin \beta_{1}-\sin \theta\right) & \frac{l}{4}\left(3 \sin \beta_{1}-\cos \theta\right)
\end{array}\right]^{\mathrm{T}}, \\
& \boldsymbol{r}_{7}=\boldsymbol{r}_{8}=\left[\begin{array}{lll}
0 & l \kappa+l \cos \beta_{1} & l \lambda+l \sin \beta_{1}
\end{array}\right]^{\mathrm{T}} .
\end{aligned}
$$

Based on Eqs. (7)-(13), the following equations can be obtained:

$$
\begin{aligned}
& \ddot{\boldsymbol{r}}_{1}=\left[\begin{array}{lll}
0 & 0 & 0
\end{array}\right]^{\mathrm{T}}, \\
& \ddot{\boldsymbol{r}}_{2}=\left[\begin{array}{ccc}
0 & \frac{3}{4} u_{1}+\frac{1}{4} u_{2} & \frac{3}{4} v_{1}+\frac{1}{4} v_{2}
\end{array}\right]^{\mathrm{T}} \text {, } \\
& \ddot{\boldsymbol{r}}_{3}=\left[\begin{array}{lll}
0 & u_{1}+u_{2} & v_{1}+v_{2}
\end{array}\right]^{\mathrm{T}} \text {, } \\
& \ddot{\boldsymbol{r}}_{4}=\left[\begin{array}{ll}
0 & u_{1}+\frac{1}{4} u_{2}-\frac{1}{4} v_{3} \quad v_{1}+\frac{1}{4} v_{2}-\frac{1}{4} u_{3}
\end{array}\right]^{\mathrm{T}} \text {, } \\
& \ddot{\boldsymbol{r}}_{5}=\left[\begin{array}{lll}
0 & u_{1}-v_{3} & v_{1}-u_{3}
\end{array}\right]^{\mathrm{T}} \text {, } \\
& \ddot{\boldsymbol{r}}_{6}=\left[\begin{array}{lll}
0 & \frac{3}{4} u_{1}-\frac{1}{4} v_{3} & \frac{3}{4} v_{1}-\frac{1}{4} u_{3}
\end{array}\right]^{\mathrm{T}}, \\
& \ddot{\boldsymbol{r}}_{7}=\ddot{\boldsymbol{r}}_{8}=\left[\begin{array}{lll}
0 & u_{1} & v_{1}
\end{array}\right]^{\mathrm{T}} \text {, }
\end{aligned}
$$

where

$$
\begin{aligned}
& u_{1}=\frac{\partial^{2}\left(l \cos \beta_{1}\right)}{\partial t^{2}}=-l\left[\omega^{2} \cos \left(\theta+30^{\circ}\right)+\alpha \sin \left(\theta+30^{\circ}\right)\right], \\
& v_{1}=\frac{\partial^{2}\left(l \sin \beta_{1}\right)}{\partial t^{2}}=-l\left[\omega^{2} \sin \left(\theta+30^{\circ}\right)-\alpha \cos \left(\theta+30^{\circ}\right)\right], \\
& u_{2}=\frac{\partial^{2}\left(l \cos \beta_{2}\right)}{\partial t^{2}}=-l\left[\omega^{2} \cos \left(\theta-30^{\circ}\right)+\alpha \sin \left(\theta-30^{\circ}\right)\right],
\end{aligned}
$$

$$
\begin{gathered}
v_{2}=\frac{\partial^{2}\left(l \sin \beta_{2}\right)}{\partial t^{2}}=-l\left[\omega^{2} \sin \left(\theta-30^{\circ}\right)-\alpha \cos \left(\theta-30^{\circ}\right)\right], \\
u_{3}=\frac{\partial^{2}(l \cos \theta)}{\partial t^{2}}=-l\left[\omega^{2} \cos \theta+\alpha \sin \theta\right], \\
u_{3}=\frac{\partial^{2}(l \cos \theta)}{\partial t^{2}}=-l\left[\omega^{2} \cos \theta+\alpha \sin \theta\right] .
\end{gathered}
$$

Recall that $\omega=\omega_{0}+\alpha t$, where $\omega_{0}$ is the initial angular speed, $\alpha$ is the acceleration of angular speed, and $t$ denotes time.

Let $l=100 \mathrm{~mm}, m_{1}=2 \mathrm{~kg}, m_{2}=3 \mathrm{~kg}$, substituting Eqs. (7)-(20) in to Eq. (4), $y_{\text {zmp }}$ can be expressed as follows:

$$
\begin{aligned}
y_{\text {zmp }}= & -\left(274 \alpha-14400 \sin \theta-307 \omega_{0}^{2} \sin \theta+14400 \sqrt{3}\right. \\
& +14400 \sqrt{3} \cos \theta+307 \alpha \cos \theta+59 \sqrt{3} \alpha \sin \theta \\
& \left.+59 \sqrt{3} \omega_{0}^{2} \cos \theta\right) /\left(480 \omega_{0}^{2} \cos \theta+480 \alpha \sin \theta\right. \\
& \left.+1440 \sqrt{3} \omega_{0}^{2} \sin \theta-1440 \sqrt{3} \alpha \cos \theta-288000\right) .
\end{aligned}
$$

According to above equations, the effects of the input parameters $\left(\omega_{0}\right.$ and $\left.\alpha\right)$ on the ZMP are discussed. Considering the condition of constant angular velocity $\left(\alpha=0\left(^{\circ}\right) / \mathrm{s}^{2}, \omega_{0}=\{0,180,270\}\left(^{\circ}\right) / \mathrm{s}\right)$, the solid line represents the centroid curve when $\omega_{0}=0\left({ }^{\circ}\right) / \mathrm{s}$. Figure 4 (a) illustrates the corresponding ZMP curves when the sixangular robot moves from the initial state to the left side. When $\omega_{0}=180\left(^{\circ}\right) / \mathrm{s}$, and $\theta=109^{\circ}$, the $y_{\text {zmp }}$ will be less than 0 , and the robot will roll about point $O$ to the left side. This angle $\left(\theta=109^{\circ}\right)$ will be called the critical angle of sixangular robot. Similarly, when $\omega_{0}=270\left({ }^{\circ}\right) / \mathrm{s}$ and $\theta=$ $98.3^{\circ}$, the robot begins to roll. Considering different angular acceleration speeds, the corresponding ZMP curves are shown in Fig. 4(b). The critical angles of the robot to roll are $\theta=114.3^{\circ}$ and $\theta=111.7^{\circ}$, wherein the greater speed the robot has, the easier the robot rolls.

Similarly, the $y_{\mathrm{zmp}}$ of eight-angular robot can be expressed as follows:

$$
\begin{aligned}
& y_{\mathrm{zmp}}=\left(\begin{array}{l}
1611 \alpha-27 \alpha \sin 2 \theta+192 \omega_{0}^{2} \cos \theta \\
-1344 \omega_{0}^{2} \sin \theta+14400 \sqrt{2} \alpha+57600 \sqrt{2} \\
-27 \omega_{0}^{2} \cos 2 \theta+81 \omega_{0}^{2} \sin 2 \theta+57600 \sqrt{2} \cos \theta \\
+1344 \alpha \cos \theta-57600 \sqrt{2} \sin \theta+192 \alpha \sin \theta \\
-81 \omega_{0}^{2}-81 \alpha \cos 2 \theta
\end{array}\right) \\
& /\left[1520\left(-\omega_{0}^{2} \sin \beta_{3}+\alpha \cos \beta_{3}+100\right)\right],
\end{aligned}
$$

where $\beta_{3}=\theta+45^{\circ}$.

The ZMP curves of eight-angular robot are plotted in Fig. 5. When $\omega_{0}=180\left(^{\circ}\right) / \mathrm{s}$ and $\alpha=0\left(^{\circ}\right) / \mathrm{s}^{2}$, the critical angle of the eight-angular robot is $81.7^{\circ}$, which is smaller 


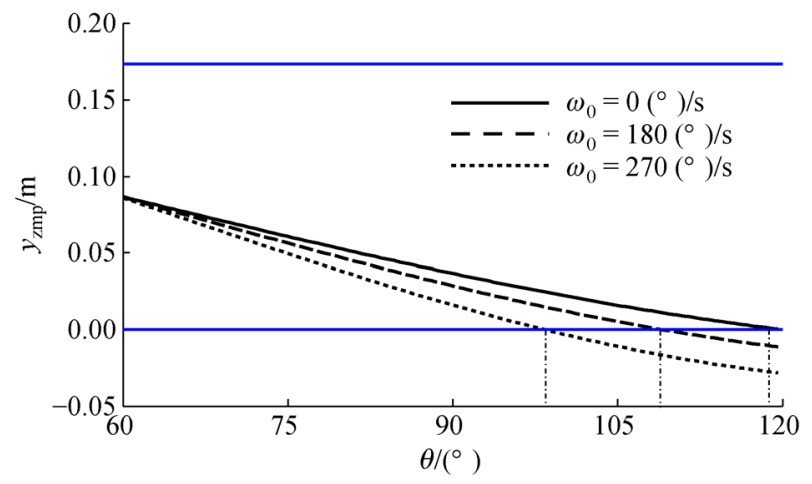

(a)

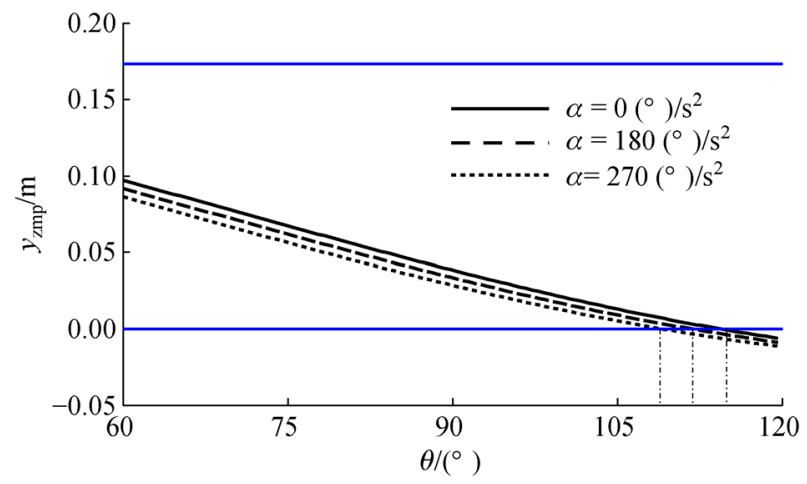

(b)

Fig. 4 ZMP curves of six-angular robot with different (a) angular and (b) acceleration speeds.

than the angle of the six-angular robot $\left(109.1^{\circ}\right)$. Using the same input parameters, the eight-angular robot needs smaller deformation to make the robot roll. Accordingly, the eight-angular robot is easier to roll than the six-angular robot.

\section{Passive rolling locomotion}

In this section, a novel passive rolling mode is developed. Without using the driven motor, the mechanism of our robot can retain a stable state at some positions or roll step by step along the slope. We will use the six-angular robot as an example to describe the passive rolling locomotion.

\subsection{Static stability state}

Figure 6 illustrates a stable state of the mechanism on a slope. To retain this static stability state, the following conditions are required:

1) No sliding motion with respect to the slope;

2) No deforming motion caused by its gravity; and

3) The centroid projection of the mechanism is in its supporting area.

For the first condition, the static friction force needs to be large enough. Static friction force can be increased by adding the high friction factor material (such as rubber) on

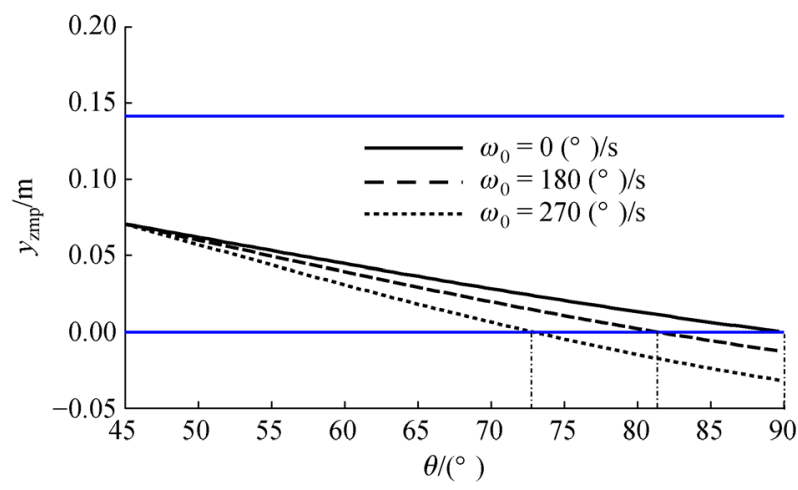

(a)

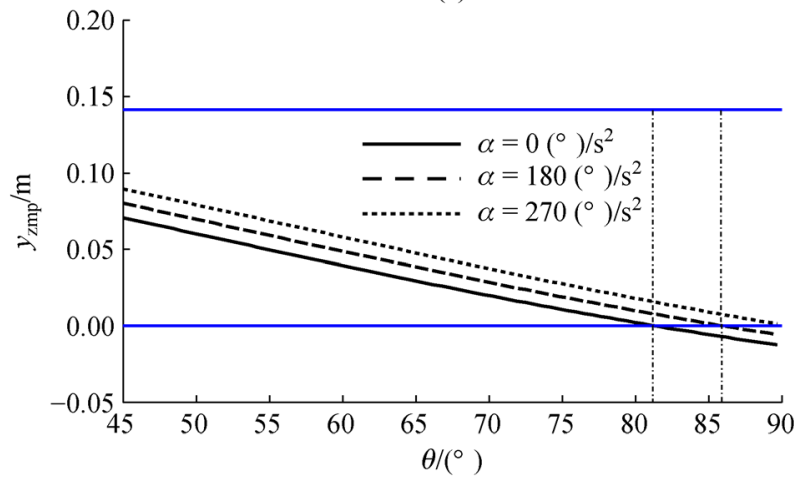

(b)

Fig. 5 ZMP curves of eight-angular robot with different (a) angular and (b) acceleration speeds.

the areas in contact. For the second and third conditions, they are the static stability problems that can be analyzed by its geometric configurations.

As shown in Fig. 6, recall that the $\mathrm{COG}$ of the mechanism is the position of the geometric center. If the mechanism is stable, the COG direction will be along line $L A$, and line $L A$ will be vertical with respect to the ground. Owing to the parallelogram configurations, lines $B C$ and $O K$ will be vertical with respect to the ground either. As a result, two branch links ( $A E I$ and $C G K$ ) are balanced on this slope. This state is called a self-balance position of the robot on a slope. According to this feature, using the angle of the slope $(\varphi)$, the balancing angle $\theta_{0}$ (the angle between $A L$ and $K L)$ at this moment can be expressed as $\theta_{0}=\varphi+$ $60^{\circ}$.

\subsection{Passive rolling mode}

According to the self-balance position, if the angle between the two branch links is $\theta>\theta_{0}$, the projection of COG will move to point $O$ but cannot exceed it. In this case, the robot will deform to left and stand on the slope. However, if $\theta<\theta_{0}$, the projection of COG will move to the right side of point $O$. Once it moves out of line $O B$, the robot will lose stability and start to roll. The angle of $\theta$ at this moment can be calculated as follows. 


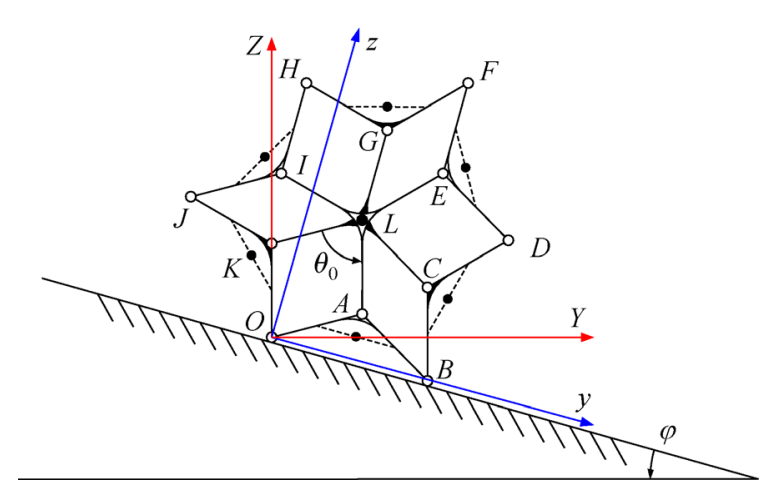

Fig. 6 Static stability state of the six-angular robot.

As shown in Fig. 6, two coordinate systems $(O-X Y$ and $o-x y$ ) are established at point $O$. The first one is the global system on the slope, whereas the second one is a local system of the robot. According to the coordinate transformation, the COG of the robot $(\boldsymbol{R})$ and position of $\left(\boldsymbol{R}_{B}\right)$ in the $O-X Y$ system can be written as follows:

$$
\begin{aligned}
& \boldsymbol{R}=\left[\begin{array}{c}
0 \\
\left(l \kappa+l \cos \beta_{1}\right) \cos \varphi+\left(l \lambda+l \sin \beta_{1}\right) \sin \varphi \\
\left(l \lambda+l \sin \beta_{1}\right) \cos \varphi-\left(l \kappa+l \cos \beta_{1}\right) \sin \varphi
\end{array}\right], \\
& \boldsymbol{R}_{B}=\left[\begin{array}{lll}
0 & 2 l \kappa \cos \varphi & -2 l \kappa \sin \varphi
\end{array}\right]^{\mathrm{T}} .
\end{aligned}
$$

Recall that $\beta_{1}=\theta+45^{\circ}$. Then, let $R_{y}=R_{B}, y$, which leads to

$$
\begin{aligned}
& \cos \left(\theta+30^{\circ}\right) \cos \varphi+\sin \left(\theta+30^{\circ}\right) \sin \varphi \\
& =\frac{\sqrt{3}}{2} \cos \varphi-\frac{1}{2} \sin \varphi .
\end{aligned}
$$

According to the kinematics, the angle of $\varphi$ needs to be large enough. Otherwise, $R_{y}$ is always smaller than $R_{B, y}$, which means the robot is unable to roll on the slope. If $\varphi$ is large enough, then the critical angle (denoted by $\theta_{1}$ ) for rolling can be calculated through Eq. (25).
The detail of the passive rolling process is described as follows:

(a) Figure 7(a) illustrates the robot at its initial position with link $O A B$ supporting on the slope. Owing to gravity, the deformation movement occurs when $\theta<\theta_{0}$, and the robot will begin to move to the right side.

(b) As shown in Fig. 7(b), when $\theta$ is reduced to the critical angle $\theta_{1}$, the robot will roll around the point $B$.

(c) As shown in Fig. 7(c), after the rolling step, link $B C D$ is the supporting link. Owing to inertial force, the robot will continue to deform and roll to the right side. In this case, the robot will roll passively along the slope with its own gravity. This rolling process is called the passive rolling mode. Our passive rolling mode can be viewed as a kind of passive walking caused by its shape morphing strategies and gravity $[18,19]$.

\section{Prototypes and experiments}

In this section, two prototypes were manufactured (referring to Fig. 8) to test the rolling locomotion. One servo motor, controller, and battery are mounted in the middle of the prototype, which also keep the center of mass close to the geometric center. Table 1 provides the specifications of prototypes. A wireless control method is used to drive our robots. As shown in Figs. 9 and 10, the active rolling experiments of the two robots were successfully performed on the horizontal ground to show their rolling capability (see the video found from the Electronic Supplementary Material 1).

The passive rolling mode of our mechanisms is demonstrated on a slope by two prototypes without any motor and controller. As shown in Figs. 11 and 12, at the initial state, both mechanisms are at their self-balance states. Once given a slight contact to break the balance position, the passive rolling locomotion will be generated. The experiments are successfully realized by their gravity. The slope angles of the six-angular and the eight-angular robots are set at $18.0^{\circ}$ and $11.3^{\circ}$, respectively. The screen

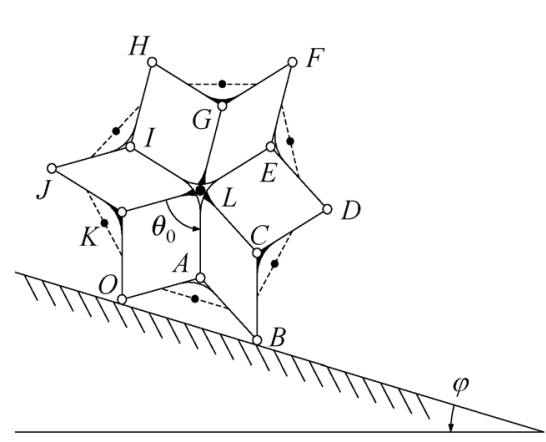

(a)

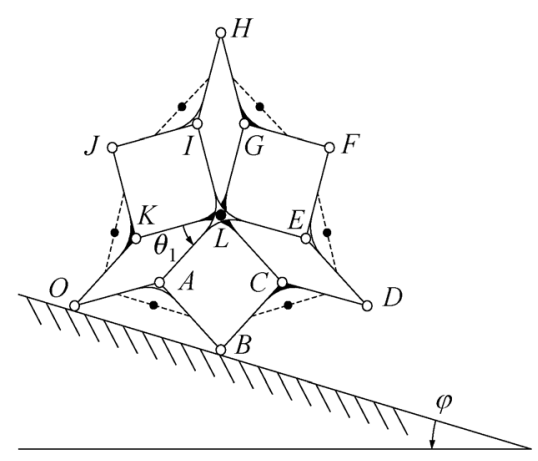

(b)

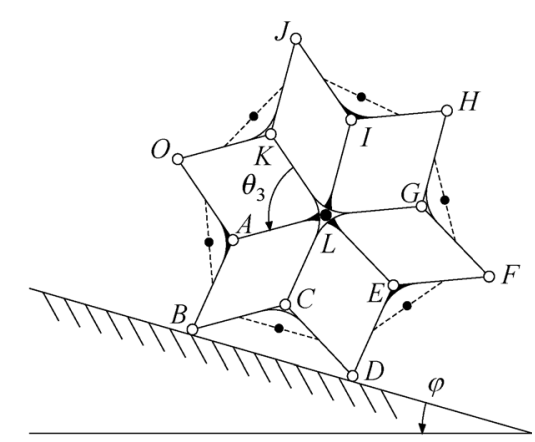

(c)

Fig. 7 Passive rolling mode. (a) About to deform; (b) about to tumble; and (c) after the tumbling motion. 

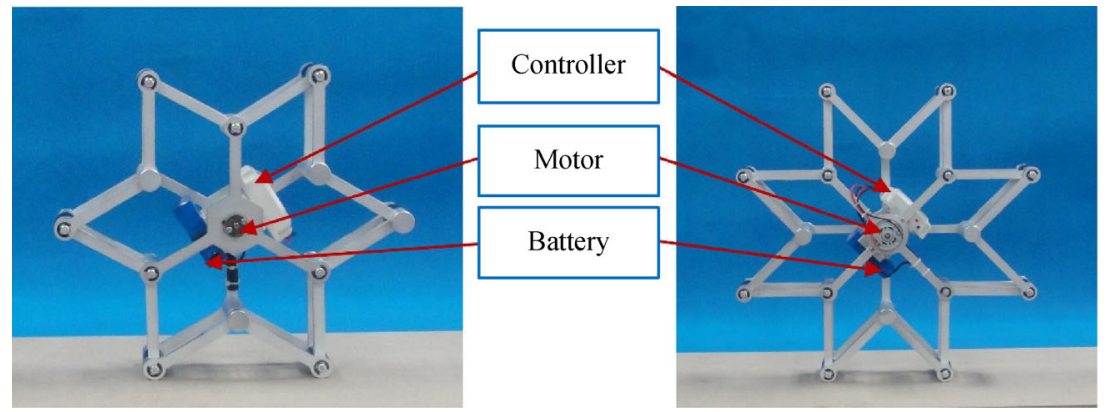

Fig. 8 Prototypes of the six- and eight-angular robots.

Table 1 Specifications of prototypes

\begin{tabular}{lc}
\hline Robot & Weight $/ \mathrm{kg}$ \\
\hline Six-angular robot & 2.5 \\
Eight-angular robot & 3.8 \\
\hline &
\end{tabular}

(a)

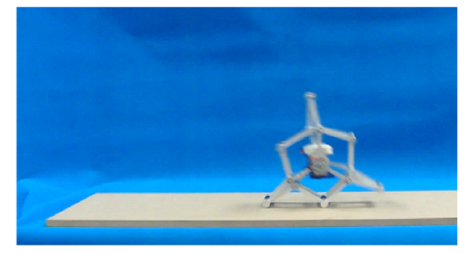

(b)

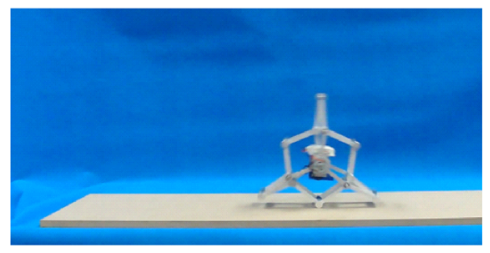

(c)

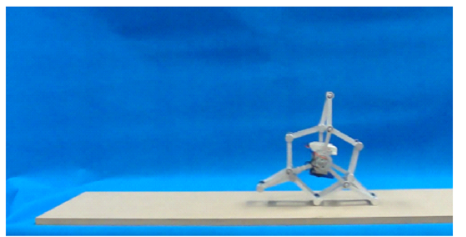

(d)

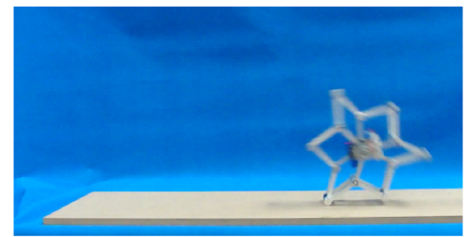

(e)

Fig. 9 Rolling experiments of the six-angular linkage.

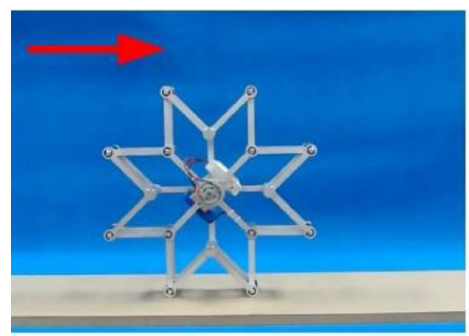

(a)

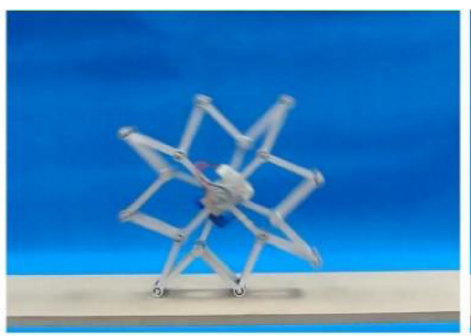

(b)

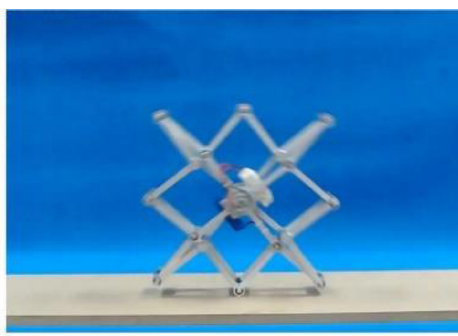

(c)

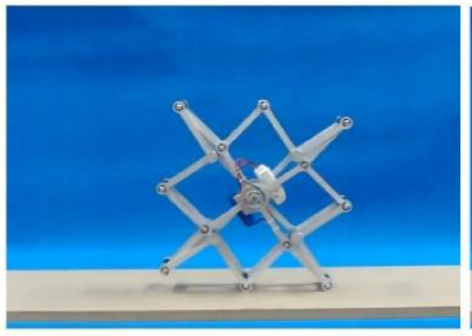

(d)

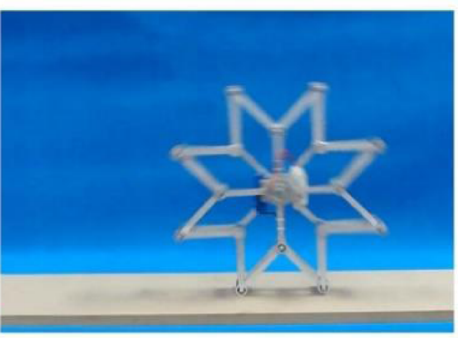

(e)

Fig. 10 Rolling experiments of the eight-angular linkage. 


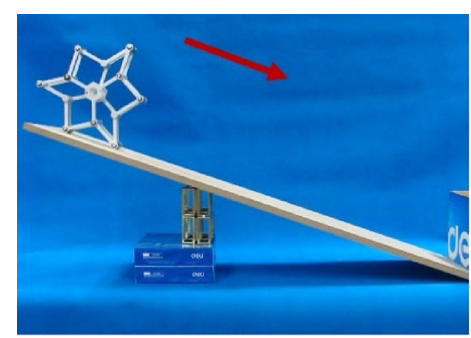

About to deform

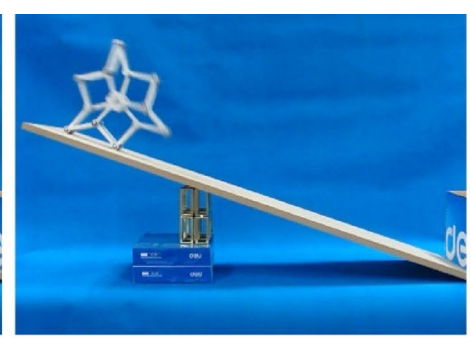

About to tumble

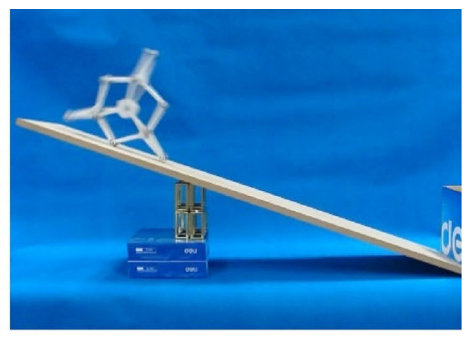

After the tumbling motion

Fig. 11 Passive rolling experiment of the six-angular linkage.

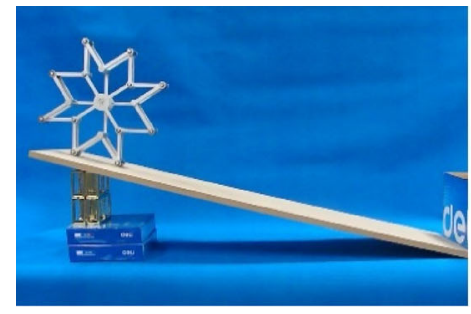

About to deform

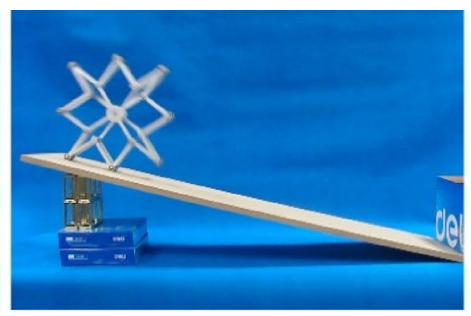

About to tumble

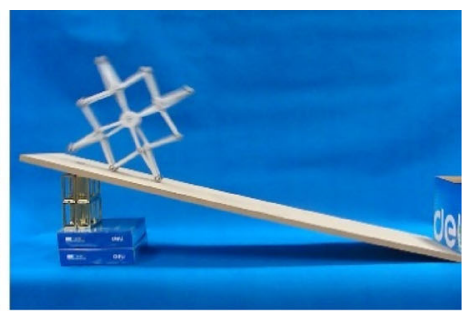

After the tumbling motion

Fig. 12 Passive rolling experiment of the eight-angular mechanism.

captures in Figs. 11 and 12 show the key steps corresponding to our analysis in Section 4. The full process of the two passive rolling experiments can be seen in the Electronic Supplementary Material 2.

\section{Conclusions}

In this study, two kinds of rolling robots are constructed by planar expandable linkages with a single DOF. Each robot has an outer concave polygonal loop and multiple parallelogram loops for ease of rolling. Using a single DOF, the robots are able to deform their shape and perform a fast dynamic rolling process along a straight line. The effects of input angular and acceleration speeds to the rolling capability were analyzed on the basis of ZMP method. In addition, a novel passive rolling mode was developed to allow the robots to stand and roll on a slope. Two prototypes were manufactured to verify the design. The active and passive rolling experiments have been successfully carried out.

In our future research, the following conditions will be considered: First, some soft materials or joints will be used to build a soft rolling robot; second, the dynamic passive rolling mode will be further analyzed; third, this work will be extended to the spatial expandable linkages to allow the robot to roll and switch its directions with a single DOF.

Acknowledgements This work was supported by the Fundamental Research Funds for the Central Universities (Grant No. 2019JBZ109).
Electronic Supplementary Material The supplementary material can be found in the online version of this article (https://doi.org/10.1007/s11465020-0585-3) and is accessible to authorized users.

Open Access This article is licensed under a Creative Commons Attribution 4.0 International License, which permits use, sharing, adaptation, distribution and reproduction in any medium or format, as long as you give appropriate credit to the original author(s) and the source, provide a link to the Creative Commons licence, and indicate if changes were made.

The images or other third party material in this article are included in the article's Creative Commons licence, unless indicated otherwise in a credit line to the material. If material is not included in the article's Creative Commons licence and your intended use is not permitted by statutory regulation or exceeds the permitted use, you will need to obtain permission directly from the copyright holder.

To view a copy of this licence, visit http://creativecommons.org/licenses/ by/4.0\%.

\section{References}

1. Armour R H, Vincent J F V. Rolling in nature and robotics: A review. Journal of Bionics Engineering, 2006, 3(4): 195-208

2. Henschel J. Feature creature-The golden wheel spider. Gobabeb Times, 2005, 1: 3

3. Caldwell R L. A unique form of locomotion in a stomaopodbackward somersaulting. Nature, 1979, 282(5734): 71-73

4. Phipps C C, Shores B E, Minor M A. Design and quasi-static locomotion analysis of the rolling disk biped hybrid robot. IEEE Transactions on Robotics, 2008, 24(6): 1302-1314

5. Sproewitz A, Moeckel R, Maye J, et al. Learning to move in modular robots using central pattern generators and online optimization. International Journal of Robotics Research, 2008, 
27(3-4): 423-443

6. Shi R D, Zhang X L, Tian Y B, et al. A CPG-based control method for the rolling locomotion of a desert spider. In: Proceedings of IEEE Workshop on Advanced Robotics and its Social Impacts. Shanghai: IEEE, 2016, 243-248

7. Tian Y B, Yao Y A. Dynamic rolling analysis of triangularbipyramid robot. Robotica, 2015, 33(4): 884-897

8. Liu C H, Yao Y A, Li R M, et al. Rolling 4R linkages. Mechanism and Machine Theory, 2012, 48: 1-14

9. Yamawaki T, Mori O, Omata T. Nonholonomic dynamic rolling control of reconfigurable $5 \mathrm{R}$ closed kinematic chain robot with passive joints. In: Proceedings of IEEE International Conference on Robotics and Automation. Taipei: IEEE, 2003, 4054-4059

10. Wang Y, Wu C, Yu L, et al. Dynamics of a rolling robot of closed five-arc-shaped-bar linkage. Mechanism and Machine Theory, 2018, 121: 75-91

11. Sastra J, Chitta S, Yim M. Dynamic rolling for a modular loop robot. International Journal of Robotics Research, 2009, 28(6): 758-773

12. Patel J, Ananthasuresh G K. A kinematic theory for radially foldable planar linkages. International Journal of Solids and Structures, 2007, 44(18-19): 6279-6298

13. Lu S N, Zlatanov D, Ding X L, et al. A new family of deployable mechanisms based on the Hoekens linkage. Mechanism and Machine Theory, 2014, 73: 130-153

14. Wohlhart K. Cyclic polyhedra and linkages derived therefrom. Mechanism and Machine Theory, 2017, 108: 142-159

15. Gogu G. Mobility of mechanisms: A critical review. Mechanism and Machine Theory, 2005, 40(9): 1068-1097

16. Kim J, Chung W K, Youm Y, et al. Real-time ZMP compensation method using null motion for mobile manipulators. In: Proceedings of IEEE International Conference on Robotics and Automation, Washington: IEEE, 2012, 1967-1972

17. Takanishi A, Tochizawa M, Takeya $\mathrm{T}$, et al. Realization of dynamic biped walking stabilized with trunk motion under known external force. In: Proceedings of the 4th International Conference on Advanced Robotics. Columbus: IEEE, 1989, 299-310

18. Inoue R, Asano F, Tanaka D, et al. Passive dynamic walking of combined rimless wheel and its speeding-up by adjustment of phase difference. In: Proceedings of IEEE/RSJ International Conference on Intelligent Robots and Systems. San Francisco: IEEE, 2011, 2530

19. Wisse M, Keliksdal G, Frankenhyyzen J, et al. Passive-based walking robot. IEEE Robotics \& Automation Magazine, 2007, 14(2): 52-62 